\title{
La posteridad sociológica de Auguste Comte: Lo normal y lo patológico en Durkheim
}

\author{
Michel BOURDEAU \\ Institut d'Histoire des Sciences et des Techniques, (CNRS-Paris1-ENS) \\ bourdeau@ehess.fr
}

Recibido: 10.02 .2008

Aceptado: 04.11.2008

Si Comte es el «padrino» de la sociología, en el sentido de que fue él quien dio nombre a la disciplina, habrá que convenir que esta última se ha construido esencialmente de espaldas a él, cuando no directamente contra él. Esta es, al menos, la idea dominante hoy en día. Sin embargo, en lo que a Francia se refiere, esto no fue siempre así. Al menos hasta los años cincuenta, y aunque le citasen raramente, todos los durkheimianos conocían perfectamente la obra del autor del Cours $^{1}$. Sin embargo, dado que el enfoque dominante en aquella época se nos ha vuelto completamente extraño, creo que la mejor manera de hablar sobre Comte a los sociólogos es refiriéndose no a su propia sociología, sino a lo que podría denominarse su posteridad sociológica y, en concreto a Durkheim.

La elección de Durkheim para explicar la influencia de Comte sobre el posterior devenir de la disciplina tiene fácil explicación. Es cierto que entre Comte y el autor del Suicide existen eslabones intermediarios, como esa primera Société de Sociologie fundada por Émile Littré en una época en la que, tras la derrota de 1870, Francia se cuestionaba si la opción republicana era o no la más adecuada. Sin embargo, la vida de la Société fue efímera y no produjo ninguna contribución significativa a la causa sociológica (Yamashita, 1995: 83-115 y Heilbron, 2007: 307-331), con lo que Durkheim ha sido considerado, con razón, como la figura fundamental en el proceso de institucionalización de la sociología francesa como disciplina universitaria. Al mismo tiempo, también es comúnmente admitido que Comte jugó un papel fundamental en la formación del pensamiento

\footnotetext{
${ }^{1}$ Ver, por ejemplo, la selección de los textos de Comte publicada por R. Hubert (1927) en la colección Les grands philosophes, Rasmussen, Paris, 1927 o el artículo de G. Davy (1949). A este respecto, es necesario recordar que durante la primera mitad del siglo pasado la sociología no tenía, al menos desde el punto de vista institucional, el status de disciplina verdaderamente independiente en las universidad. Todavía en la época de la generación de Lévy-Strauss o de Aron, la mayoría de los sociólogos tenían una formación filosófica.
} 
de Durkheim ${ }^{2}$. En este sentido, si bien es verdad que se suele citar más a menudo a Spencer como antecesor del sociólogo francés, esto se debe a razones puramente coyunturales y al hecho de que la popularidad del pensador inglés lo ha convertido en una referencia casi obligada ${ }^{3}$. Pese a ello, al menos en lo que se refiere al fondo, la realidad es que hay razones más que suficientes para pensar que las referencias a Comte, ya sean explícitas o no, son mucho más importantes. Sin embargo, y precisamente por ese carácter muchas veces implícito, esa influencia no puede ser considerada únicamente a la luz de aquellos pasajes en los que Durkheim cita a Comte. No en vano, una ley muy conocida estipula que lo mejor para matar al padre es encomendarse al abuelo. Esto era ya válido para el propio Comte quien, negándose a reconocer su deuda intelectual hacia Saint-Simon, consideraba a Condorcet como su predecesor inmediato en la fundación de la ciencia social. Del mismo modo, Durkheim tendió a minimizar la importancia de Comte, hasta el punto de presentarle como un simple discípulo de Saint-Simon. Es precisamente esta cuestión la que hace difícil rastrear en ciertos temas o conceptos durkheimianos algunos préstamos más o menos directos de Comte (especialmente hoy en día cuando los sociólogos ignoran casi por completo a este último).

Para quien se interese por la metodología, la cuestión de las relaciones entre lo normal y lo patológico será probablemente la más interesante de las que voy a analizar en este artículo. Así, como mostraré en la cuarta sección, el capítulo consagrado a esta cuestión en Les règles de la méthode sociologique (1895) tiene algo de sorprendente para el lector contemporáneo. Así, la idea de que pueda existir una «patología social» nos parece extraña hoy en día. Al fin y al cabo, y aunque los sociólogos se interesan por las normas, lo cierto es que dicha idea se nos presenta completamente desligada de la noción de «lo patológico», un término desparecido del vocabulario sociológico. En este sentido, la existencia de este capítulo nos recuerda que el universo conceptual de Durkheim no es en absoluto el nuestro y que si la distinción entre lo normal y lo patológico era evidente para el autor del Suicide, esto se debía simplemente a que pensaba en un marco que Comte había contribuido a construir. Tanto aquí como en el caso precedente, veremos como Durkheim se apropia de esta división y la trabaja de tal manera que el resultado es completamente extraño al propio Comte.

Sin embargo, como se verá en la tercera sección, el diálogo crítico entre Durkheim y Comte había comenzado dos años antes, en La división du travail social. Tal es así que esta tesis doctoral de 1893 puede ser interpretada como una refutación de una de las tesis centrales del positivismo. En efecto, Durkheim intenta demostrar que el fundador del positivismo se había equivocado en su in-

2 «La originalidad de Durkheim reside ampliamente en el hecho de que consiguió transformar un principio de Comte en un programa eficaz de investigación»: J. Heilbron (1993:65). Ver igualmente M. Gane (1997: 31-38) y J.-M. Berthelot, (2002).

${ }^{3}$ Sobre la gran operación de marketing a la que dio lugar la publicación de las obras de Spencer, ver D. Becquemont et L. Muchielli (1998) 
terpretación de la división del trabajo, y que había sido incapaz de ver que existen dos formas de solidaridad. Así, si es cierto que los progresos en la división del trabajo destruyen la solidaridad mecánica, no se puede decir lo mismo de la solidaridad orgánica. La crítica es fundamental: los argumentos que Durkheim presenta son sólidos y la política positiva se encuentra amenazada en su propio principio.

Antes de examinar estas dos cuestiones, es conveniente hacer algunas aclaraciones con respecto a la sociología de Comte (ver primera sección). Esta es la razón por la que he dividido mi exposición en tres partes seguidas de una conclusión en la que añadiré algunas cuestiones sobre la posición enciclopédica de la sociología, es decir, sobre sus relaciones con las dos disciplinas que la rodean en la clasificación de las ciencias: la biología y la moral. También en este caso Durkheim retuvo la lección del Cours, lección que, nuevamente, reinterpretó a su manera.

\section{UN BREVE REPASO DE LAS NOCIONES ELEMENTALES DE LA SOCIOLOGÍA DE COMTE}

Para un sociólogo actual, la dificultad de reconocerse en la sociología de Comte consiste, sobre todo y en primer lugar, en que para este último la ciencia sociológica tenía una doble función que constituye la conclusión fundamental del Cours: la ciencia social no es simplemente una ciencia entre otras, sino que es la ciencia destinada a ocupar la presidencia del escalafón enciclopédico, tarea que hasta ese momento habían desempeñado las matemáticas. El punto de vista humano, es decir el social, es el único capaz de comprender el conjunto de los fenómenos. En particular, y dado que toda ciencia es un hecho social, la actividad científica en su conjunto depende de la sociología, que de este modo se convierte en la encargada de coordinar la marcha general del saber. La función en la que piensa Comte es la misma que hasta ese momento había desempeñado, con mayor o menor acierto, la filosofía. Es en este contexto en el que, en la lección 58 del Cours, Comte afirmará: «El punto de vista sociológico es, de ahora en adelante y en todos los ámbitos, el único verdaderamente filosófico» ${ }^{4}$. Durkheim comprendió perfectamente que estas pretensiones hegemónicas ponían en riesgo la posibilidad de constituir una ciencia social como disciplina autónoma. Para garantizar su cientificidad, la emergente sociología debía desarrollar una única función y contentarse con ser una ciencia como las demás, algo que para Durkheim era más que suficiente.

Desde luego, no se puede decir que Comte desatendiese este segundo aspecto. Así, las lecciones del Cours permiten hacerse una idea bastante precisa de lo que Comte entendía por la ciencia de los hechos sociales. Utilizando una dis-

4 Ver : M. Bourdeau (2004) : «L'idée de point de vue sociologique », Cahiers internationaux de sociologie, CXVII (2004), p. 226-238, así como el trabajo clásico de J. Lacroix (1956) 
tinción que Comte había tomado prestada de su amigo el biólogo Blainville y que a nosotros nos resulta familiar gracias a la mecánica, Comte divide la sociología en dos partes: la estática social (statique sociale) o teoría del orden, y la dinámica social (dynamique sociale) o teoría del progreso. Una vez más, el aparato conceptual de Comte difiere notablemente del nuestro: su interés fundamental se centra en la dinámica, tal y como demuestra el lugar primordial que ocupa la ley de los tres estados en el positivismo. Sin embargo, hoy en día el determinismo histórico que está asociado a dicha ley nos parece sospechoso y típico, como sucede con Marx, de una cierta filosofía de la historia propia del siglo XIX. Pese a ello, es importante recordar que en las grandes lecciones históricas del Cours abundan los análisis de una gran sutileza que, todavía hoy, justifican plenamente su lectura ${ }^{5}$.

Sea como fuere, lo más interesante para nosotros se encuentra sin duda en la estática $^{6}$. Dicha estática gira alrededor de la idea de «consenso», que Comte toma de la medicina. La vida es consenso, es decir armonía entre las diferentes funciones de un organismo y también de la sociedad ${ }^{7}$, sociedad en la que hay que conciliar la independencia y la colaboración, la separación de los oficios y la combinación de esfuerzos (Comte, 1929, II: 293). A partir de este principio fundamental, Comte deduce que la vida social reposa sobre la confianza: «Cada día, por una continuación necesaria de la gran subdivisión actual del trabajo humano, cada uno de nosotros hace espontáneamente reposar, en varios aspectos, el mantenimiento de su propia vida sobre la aptitud y la moralidad de una multitud de agentes prácticamente desconocidos, cuya estupidez o perversidad podría afectar gravemente a masas a menudo muy extensas» (Comte, 1975, II: 279; cf. Comte, 1970: 325-327).

A diferencia de la dinámica, la estática describe las características comunes a todas las sociedades. Para ver en esta teoría de la estructura social una anticipación del estructuralismo es necesario suponer que dicha teoría ignora el tiempo, lo que no es cierto en el caso de Comte. Así, para él, los lazos humanos se tejen a lo largo de dos grandes ejes, la solidaridad y la continuidad. El primero se extiende a través del espacio y, delante de nuestros ojos, podemos percibirlo de manera inmediata. La división del trabajo ofrece probablemente el ejemplo más característico al respecto y, así por ejemplo, hoy en día nadie ignora que aquello que acontece en la otra punta del plantea tiene efectos directos sobre su vida cotidiana. El segundo se refiere al tiempo y a la sucesión de generaciones. Dado que el pasado y el futuro tienen en común la propiedad de no ser presente, es ne-

${ }^{5}$ Sobre la cuestión, siempre de actualidad, de las relaciones entre la historia y la sociología, ver: Fr. Chazel (2004)

${ }^{6}$ A este respecto, T. Parsons no se equivoca al retomar el capítulo correspondiente del Cours en su recopilación Theories of Society, New York, Glenncoe Press, 1961.

${ }^{7}$ Uno de los tres temas fundamentales de Durkheim en el discurso de apertura de la cátedra de ciencias sociales de la universidad de Burdeos en 1888 es la insistencia en el «consensus universal que caracteriza los fenómenos que rigen los cuerpos vivos y que la vida social manifiesta en su más alto grado», citado en el mencionado artículo de J. M. Berthelot, nota 2, p. 189. 
cesario un esfuerzo para representarlos. Si no es cultivado, el recuerdo se desvanece, y esta es la razón por la que luchar contra el olvido, cultivar la memoria, es algo fundamental si se quiere preservar la humanidad del hombre. No en vano, conocemos el papel decisivo que el culto a los muertos y la refundición del calendario jugó en la constitución de una nueva religión positivista. Sin embargo, es necesario recordar que la idea de humanidad en Comte es tan antigua como la propia sociología. A aquellos para quienes la segunda de las ciencias orgánicas no es más que un apéndice de la biología, Comte opone ese fenómeno sin parangón en la naturaleza que es «la influencia gradual y continua de unas generaciones humanas sobre otras» (Comte, 1975, II: 200). Desde 1822, Comte considera la historicidad como el hecho social fundamental, aquél al que todo se remite. La vida en sociedad, la solidaridad, no son propias del hombre. Si necesitamos una sociología es porque «la acción de unos individuos sobre otros es particularmente complicada en la especie humana debido a la acción que cada generación ejerce sobre aquella que la precede» $\left(\right.$ Comte, 1975 , I: 57) ${ }^{8}$.

\section{LA DIVISIÓN DEL TRABAJO Y LAS DOS FORMAS DE SOLIDARIDAD}

La cuestión de las relaciones de Comte con Durkheim se plantea en primer lugar a partir del problema de la división del trabajo social. En efecto, tal y como trataré de demostrar a continuación, la distinción entre los dos tipos de solidaridad, mecánica y orgánica, puede ser considerada como una respuesta directa a las doctrinas sociológicas del Cours de philosophie positive. Esto es algo que se ve ya en la propia elección del tema. Así, tanto la posición crítica con respecto a la economía, acusada de concebir la división del trabajo de una manera demasiado estrecha, como la idea de que dicha división se extiende al conjunto de la vida social son dos préstamos evidentes. A este respecto, Comte llegaba incluso al extremo de hacer remontar el origen de la división del trabajo a la biología, en la división fisiológica del trabajo en el interior de un organismo (Vatin, 2005). De esta manera, la división del trabajo está presente como condición sine qua non del progreso: progreso en la evolución de las especies, progreso en el desarrollo histórico de las sociedades. Sin embargo, Comte insistió siempre en los efectos colaterales que a la larga pueden comprometer todos los beneficios. El desarrollo de la división del trabajo va a la par con el de la especialización. A este respecto, Comte se interesa menos por el carácter repetitivo del trabajo en cadena que por sus efectos morales, presentes como una verdadera amenaza para la so-

${ }^{8}$ La mejor visión de conjunto sobre el pensamiento sociológico de Comte se encuentra en el capítulo que consagró al mismo Raymond Aron en Les grandes étapes de la pensée sociologique, Gallimard, 1958. Aron propone una interpretación de Comte como el téorico de la sociedad industrial, concepto a través del cual pretende superar la oposición entre socialismo y capitalismo. Al respecto, ver también: M. Gane (2006)

EMPIRIA. Revista de Metodología de Ciencias Sociales. N. ${ }^{\circ}$ 16, julio-diciembre, 2008, pp. 43-58. ISSN: 1139-5737 
lidaridad y los lazos sociales. Encerrado en un universo cuyo horizonte se hace cada vez más pequeño, el individuo vive cada día más replegado sobre sí mismo, indiferente al curso general del mundo. Para luchar contra este tipo de fenómenos, es necesario, considera Comte, crear una nueva instancia dedicada especialmente a llevar a consideraciones de conjunto a los espíritus con tendencia a la dispersión. Esto explica que el primer axioma de la política positiva será que no hay sociedad sin gobierno, encargado este último de poner en marcha sus capacidades para disminuir las divergencias y consolidar las convergencias. $\mathrm{Mu}$ tandis mutandis, el análisis se aplica también al dominio intelectual: al fin y al cabo, la distancia entre pasar la vida fabricando alfileres o atornillando un perno y dedicarla a clasificar una variedad de insectos o a resolver un tipo de ecuaciones es más pequeña de lo que parece. En cualquier caso, el resultado es siempre el mismo: la hipertrofia de un órgano y la atrofia de los otros. Sin dejar de reconocer la diversidad irreductible de las ciencias, Comte se preocupó de plantear la cuestión de la unidad de la ciencia - algo que en ocasiones denominará «convertir la ciencia en filosofía»—y, tal y como hemos visto, exigió a la sociología asegurar, en la vida científica, la función del gobierno en la vida social.

Durkheim comprendió perfectamente la importancia de esta cuestión para el pensamiento positivo en su conjunto. En la crítica que dirige a Comte a través de lo que en un primer momento podría parecer una cuestión de detalle (a saber: una concepción demasiado restrictiva del concepto de solidaridad), Durkheim está apuntado a toda la filosofía positiva, y especialmente al modo en que dicha filosofía pretendía articular filosofía de la ciencia y filosofía política,

Aunque A. Comte ha reconocido que la división del trabajo es una fuente de solidaridad, parece no haberse dado cuenta de que esta solidaridad es sui generis y de que es sustituida poco a poco por aquella que engendran las diferencias sociales. Esta es la razón por la que, señalando que dichas diferencias tienden a borrarse allí donde las funciones son muy especializadas, Comte vio en ese desvanecimiento un efecto mórbido, una amenaza para la cohesión social debida al exceso de especialización [Esto es algo en lo que Comte se equivocaba, puesto que] si, en algunos casos, la solidaridad orgánica no es todo lo que debería ser, esto es algo que no se debe a que la solidaridad mecánica haya perdido terreno, sino a que todas las condiciones de existencia de la primera no han sido llevadas a cabo (Durkheim, 1973:356)

Crisis económicas, quiebras, conflictos sociales, los fenómenos mórbidos señalados por Comte evidentemente existen, pero no son la consecuencia necesaria de la división del trabajo y, si los positivistas lo han creído así es porque parten de una idea inadecuada de la solidaridad. En efecto, Durkheim asocia los progresos de la división del trabajo al tránsito de las sociedades que llama de tipo «segmentario» a las de tipo «orgánico», que se corresponden con dos formas de solidaridad, mecánica en un caso y orgánica en el otro. Esta distinción le permite no tanto escapar a las críticas dirigidas a la división del trabajo, sino poner en duda alguna de las ideas directrices del positivismo. Si en su proyecto conside- 
ra todavía la posibilidad de hacer un llamamiento al estado para regular la vida económica, insiste también en señalar que este último es incapaz de asegurar el consenso si este es incapaz de realizarse por sí mismo. De la misma manera, Durkheim insiste en que la filosofía es cada día más incapaz de asegurar la unidad de las ciencias. Sobre esta última cuestión, Durkheim oscila entre dos posiciones: en algunos momentos deja entrever que hay que renunciar a la unidad y en otros considera que para garantizarla es suficiente con que «todos aquellos que cultivan la ciencia sientan que participan de una misma obra». Lo que es evidente es que, para garantizar la positividad de la sociología, Durkheim escogió quedarse únicamente con una de las dos funciones que Comte asignaba a dicha ciencia: la de convertirse en una auténtica ciencia de los hechos sociales, renunciando a la tarea de asegurar la buena marcha del conjunto del saber. En el siglo Xx, serán filósofos como O. Neurath o John Dewey quienes recordarán que la unidad de la ciencia es también un problema social ${ }^{9}$.

\section{LO NORMAL Y LO PATOLÓGICO}

Estas breves indicaciones son suficientes para mostrar la distancia que separa lo que el autor del Cours denominó durante mucho tiempo «la física social»y «la sociología» tal y como se practica hoy en día. Si dicha distancia nos parece tan grande es porque la posteridad sociológica de Auguste Comte ha pasado a menudo a través de otras vías, tal y como lo demuestra la distinción que Durkheim establece entre lo normal y lo patológico.

\section{Comte, el principio de Broussais y la teoría del orden modificable}

Los trabajos de G. Canguilhem han sentado las bases sobre esta cuestión ${ }^{10}$. Ecrits de jeunesse, Cours de philosophie positive, Système de politique positive, la cuestión de las relaciones entre lo normal y lo patológico se encuentra en cada una de las tres etapas que marcan el desarrollo del pensamiento de Comte y el «principio de Broussais», que formula la solución escogida, ocupa una posición eminente en la epistemología positiva al mismo tiempo que, a través del concepto de orden modificable, da la clave de la relación entre la teoría y la práctica.

Bajo una forma general, el principio afirma la identidad de lo normal y de lo patológico y define sus diferencias en términos cuantitativos: «Toda modifica-

\footnotetext{
9 Ver, por ejemplo, J. Dewey (1939)

10 «La identidad real de los fenómenos vitales normales y patológicos, aparentemente tan distintos y representando valores tan opuestos, se convirtió, a la largo del siglo XIX, en una especie de dogma, científicamente demostrado, cuya extensión [...] parecía garantizada por la autoridad que los biólogos y los médicos le reconocían. En Francia, ese dogma fue expuesto con intenciones bien diferentes por Auguste Comte y Claude Bernard», (Canguilhem, 1966:14)
} 
ción artificial o natural, del orden real, concierne solamente a la intensidad de los fenómenos correspondientes [...] pese a las variaciones de grado, los fenómenos conservan siempre el mismo acuerdo, todo cambio de naturaleza propiamente dicho, es decir de clase, es reconocido por tanto como contradictorio» (Comte, 1929, III: 71). El principio fue atribuido al médico francés Broussais, que fue el primero en establecerlo para los fenómenos biológicos: «El estado patológico había sido hasta entonces relacionado con leyes totalmente distintas a las que rigen el estado normal, de tal modo que el análisis de uno no podía aportar nada al conocimiento del otro. Broussais estableció que los fenómenos de la enfermedad coinciden esencialmente con los de la salud, y que sólo se diferencian por su intensidad» (Comte, 1929, I: 651). A Comte sólo le quedará generalizar este descubrimiento, aplicándolo a todas las «variaciones excepcionales, calificadas de perturbaciones en la existencia inorgánica, de enfermedades en los seres vivos y de revoluciones en la vida colectiva» (Comte, 1929, II: 432). A través de la extensión sistemática de este principio, el autor del Système venía prácticamente a reivindicar la paternidad intelectual de este principio. Por otra parte, la proximidad existente entre las dos ciencias de los seres organizados que son la biología y la sociología hacía que la extensión del principio a los fenómenos sociales fuese casi inmediata. Así, para designar el estado de anarquía en el que, en su opinión, habían caído nuestras sociedades, Comte hablará de «enfermedad occidental». Si Durkheim no le sigue en esta cuestión, lo cierto es que tampoco pone en duda la existencia de una patología social.

El orden modificable. El principio de Broussais sirve de fundamento para un concepto que permite igualmente pensar la relación entre la teoría y la práctica o, en otros términos, la acción del hombre sobre la naturaleza: nos referimos al concepto de orden modificable. En este caso, el punto de partida lo proporciona la clasificación de las ciencias. Desde las matemáticas hasta la sociología, se procede, como es de sobra conocido, desde lo más simple hasta lo más complejo, de lo más general a lo más particular. En cambio, lo que generalmente olvidamos señalar es que la complejidad va emparejada con la «modificabilidad» (modificabilité) o, si se prefiere, con la inestabilidad. Cuanto más complejo es un fenómeno, más depende su curso normal de una multiplicidad de factores cuya presencia simultánea no es en absoluto evidente, de tal manera que dicho fenómeno se verá perturbado si una de esas condiciones no es satisfecha. Esto vale para los fenómenos químicos en la medida en que se diferencian de los físicos (así por ejemplo una reacción química no se producirá más que a una determinada temperatura). Esto también vale para los fenómenos biológicos y así por ejemplo la vida no es posible más que en determinadas condiciones bien definidas, que cambian según cada especie. Las consecuencias de esta cuestión sobre la buena marcha de los experimentos son perfectamente conocidas. Sin embargo, para que tenga sentido continuar hablando de «orden» es necesario que dichas variaciones no sean ilimitadas. En definitiva, la idea de «modificabilidad» es inseparable de la de «límites de variación» que es necesario por tanto determinar. Por encima o por debajo de una cierta temperatura, de una determinada presión, 
un ser vivo muere. Es evidente que la existencia de lo patológico tiene que ser pensada en este marco: se trata de una modificación del estado normal y sabemos que a Comte le gustaba, como a muchos otros, ver en ello el equivalente natural de la experimentación.

Una vez reconocida la existencia de estas modificaciones, tiene sentido distinguir dos tipos, según cada una de ellas provenga de la naturaleza o del arte, entendido aquí como una técnica. Así por ejemplo, en nuestro sistema solar las órbitas de los planetas se ven naturalmente perturbadas por la presencia de otros planetas; en cambio, es la acción humana (el desarrollo industrial) la responsable de la destrucción de la capa de ozono. Del mismo modo, las enfermedades unas veces son naturales y otras artificiales: alguien puede ser ciego de nacimiento o se puede quedar ciego debido a una manipulación peligrosa o a una agresión. Los límites de variación sirven de este modo para definir el campo de la acción humana. Ya desde 1822, Comte había concebido el proyecto de un tratado sobre la industria, entonces llamado tratado sistemático de la acción del hombre sobre la naturaleza y esto sin duda explica su interés por la idea de «modificabilidad», que es la condición de posibilidad y de inteligibilidad de esta acción. La teoría del orden modificable y sus corolarios que son las ideas de límites de variación y de acción, no está exenta ni de originalidad ni de fuerza ${ }^{11}$. Esto concierne directamente al sociólogo, puesto que el arte político se apoya sobre la sociología de la misma manera que el arte médico lo hace sobre la biología. En política como en todo lo demás, es necesario admitir que no gobernamos los fenómenos y que lo más que podemos hacer es modificarlos para obtener un beneficio. Es importante señalar que también Durkheim asocia la reflexión sobre lo normal y lo patológico a la relación de la teoría con la práctica, de la ciencia con la acción. ${ }^{12}$ Es precisamente allí donde comienza el capítulo del que vamos a hablar a continuación.

${ }^{11}$ El último capítulo del tomo Segundo del Système de politique positive está consagrado a la «teoría positiva de los límites generales de las variaciones propias del orden humano» y sirve de transición entre la estática, que acaba de concluir, y la dinámica, que será el objeto del tomo tercero. Consciente de la novedad de sus propósitos, Comte comienza con algunas reflexiones previas destinadas a «preparar a los raciocinios poco cultivados» (pp. 426-427). Un poco más adelante, «la identidad fundamental de la salud y de la enfermedad» es presentada como constituyente «del sentido fundamental del principio de modificabilidad» (p. 457). La idea de límite no remite solamente a la de variación sino que, en la tradición matemática, remite también a la de aproximación. Así por ejemplo, nos acercamos siempre al límite pero nunca lo alcanzamos. La idea de una transgresión de los límites no tiene en esta ocasión sentido, sin embargo existe la idea de conocimiento aproximado.

${ }_{12}$ Toda vez en posesión de un criterio objetivo que permite diferenciar la salud de la enfermedad, «la ciencia se encontrará en condiciones de esclarecer la práctica permaneciendo fiel a su propio método [...] Entre la ciencia y el arte, ya no existe un abismo; sino que se pasa de una a otra sin solución de continuidad. La ciencia, es cierto, no puede descender a los hechos más que gracias a la intermediación del arte, pero el arte no es más que la prolongación de la ciencia» (Durkheim, 1998:49) 


\section{Durkheim o la puesta en marcha del concepto comtiano: norma y media}

El capítulo de las Règles que nos interesa ofrece un excelente ejemplo de la manera que tiene Durkheim de retomar los temas comtianos para modificar profundamente su contenido e incluso, estaría tentado de decir, para subvertirlos. Durkheim encontrará el criterio objetivo que necesita en la identificación de la norma y la media, lo que, en su caso, se produce a través de la estadística, tema en el que está más cerca de Quételet que de Comte ${ }^{13}$. Este último siempre condenó con vehemencia la aplicación de las matemáticas a las ciencias sociales. Si Comte acuñó la palabra sociología, fue para desmarcarse de Quételet, que había utilizado la expresión física social «en una obra donde se trata más o menos de simple estadística» (Comte, 1975, II: 15n)

En primer lugar, nos gustaría recordar brevemente el contenido de este capítulo. Después de los pasajes ya mencionado sobre las relaciones entre la ciencia y la acción, Durkheim comienza rechazando un criterio de inspiración darwiniana: diferenciar lo normal y lo patológico en base a la adaptación, la supervivencia, supondría que se sabe exactamente en qué consiste la vida y la muerte de una sociedad, lo que ciertamente no es el caso. La respuesta seleccionada se basa en una diferencia formal y por tanto objetiva: ciertas formas son generales, en el sentido de que se observan en la mayoría de los casos, mientras otras se presentan raramente,

«Llamaremos normales a los hechos que presentan formas generales y daremos a los otros el nombre de mórbidos o patológicos. Si es conveniente denominar «tipo medio» a ese ser esquemático que constituimos juntando en un todo, en una especie de individuo abstracto, las características más frecuentes en una especie con sus formas más frecuentes, se podrá decir entonces que el «tipo normal» se confunde con el «tipo medio» y que toda desviación con respecto a este patrón de salud es un fenómeno mórbido» (Durkheim, 1998:56)

Aunque esta noción sea objetiva, lo cierto es que no tiene nada de absoluto, puesto que es relativa a una especie e incluso, en el interior de dicha especie, a los diversos estados de desarrollo del individuo. No obstante, lo fundamental es que todo se reduce ahora a consideraciones estadísticas, de las que Quételet había aportado el modelo y que Durkheim había utilizado de manera masiva en su obra sobre el suicidio. Una segunda sección busca entonces transformar «esta normalidad de hecho en una normalidad de derecho» (Durkheim, 1998:59): confirmando, si se prefiere, lo bien fundado de esta definición a través de una explicación del fenómeno de generalidad sobre el que reposa. Es entonces cuando

${ }^{13}$ Durkheim había criticado a Quételet porque, en su opinión, la consciencia colectiva no era la consciencia media y, por lo tanto, el científico belga se había equivocado al definir, a través del tipo medio, el tipo colectivo que es el objeto de la sociología (ver: Durkheim, 1960: 337-338). En realidad, la idea de media se encuentra más desplazada que incluida: sirve para definir no lo social sino lo normal. 
la naturaleza de las cosas, que había sido excluida de las consideraciones precedentes, es reintroducida. Una última sección responde a quienes juzga inútilmente compleja la investigación desarrollada. Dicha investigación se justifica por la complejidad superior que caracteriza a los fenómenos sociales. Durkheim ve así la ocasión de mostrar incluso en sus consecuencias paradoxales la fecundidad de su criterio que hace aparecer el crimen como un fenómeno normal e incluso como «un factor de salud pública» (Durkheim, 1998:66).

El capítulo que acabamos de analizar ha llamado poco la atención de los especialistas, que se han contentado simplemente con señalar su carácter problemático: desde este punto de vista, su supresión no afectaría al conjunto de la obra y la argumentación presentada no estaría exenta de debilidades. Así, mientras que se alaba a Durkheim por haber liberado la sociología de la tutela de la biología, es sorprendente constatar la fuerza con la que se impone el modelo biológico ${ }^{14}$. En el presente caso, nos quedaremos sobre todo con la fuerza de la relación con Comte y con su ambigüedad. Fue el fundador del positivismo quien, invitando a pensar la sociología a través de su relación con la biología, condujo a Durkheim a ampliar a la sociología la distinción entre lo normal y lo patológico que se buscaría en vano en Quételet. Pero la deuda se acaba aquí, y el criterio propuesto por Durkheim no debe nada más al autor del Cours $^{15}$. Si la palabra estadística no aparece, el vocabulario utilizado (media, desviación) no deja ninguna duda y, de hecho, eso será precisamente lo que retendrán los sociólogos. Para la mayoría de los lectores contemporáneos, la idea de una patología social no tiene más que un interés puramente histórico, en tanto que contexto que sirvió para justificar la utilización de la estadística en sociología.

\section{CONCLUSIÓN}

Moral y sociología. Un último punto, rápidamente, antes de concluir. El tema de lo normal y de lo patológico acaba de remitirnos a la cuestión de las relaciones entre la sociología y la biología. Sin embargo, para valorizar la poste-

${ }^{14}$ Una vez definido «tipo normal» $\mathrm{y}$ «tipo medio», Durkheim continua: «Lo que el fisiologista estudia son las funciones del organismo medio, y el sociólogo no procede de otro modo». Algunas líneas más adelante, y en esta ocasión para justificar que la distinción es siempre relativa a una especie dada, Durkheim señala: «La regla no ha sido contestada en biología [...] El mismo principio se aplica a la sociología aunque muchas veces sea desconocida: (Durkheim, 1998:56). Ver Guillo (2006)

${ }^{15}$ No obstante es necesario recordar que la idea no era tan extraña a Comte como se suele decir. A pesar de la hostilidad hacia las matemáticas sociales y la teoría de las probabilidades, la idea aparece de hecho claramente formulada en: Système de politique positive, t. II, p. 431: «El tipo normal no remite más que a un estado medio, más ideal que real, alrededor del cual oscila sin cesar la existencia efectiva». El modelo no está exento de reminiscencias biológicas: «La marcha de la civilización no se lleva a cabo, a decir verdad, siguiendo una línea recta, sino a partir de una serie de oscilaciones, desiguales y variables, como sucede con la locomoción animal, alrededor de un movimiento medio que tiende siempre a predominar» (Comte, 1975, II:151)

EMPIRIA. Revista de Metodología de Ciencias Sociales. N. ${ }^{\circ}$ 16, julio-diciembre, 2008, pp. 43-58. ISSN: 1139-5737 
ridad sociológica de Comte, y en particular su influencia sobre Durkheim, también hubiera podido afrontar el problema en cierto sentido complementario de las relaciones entre la moral y la sociología. Las dos cuestiones están menos alejadas de lo que pudiera parecer. La idea de norma es evidentemente equívoca ${ }^{16}$. Pensada como media, dicha idea se sitúa en el orden de los hechos. Sin embargo, pensada como normatividad, nos remite al mundo de los valores, pasando del hecho al derecho. Durkheim se aprovechó de esta ambigüedad tal y como el ejemplo del crimen nos acaba de mostrar.

Uno de los puntos esenciales de la epistemología positivista es el de invitarnos a pensar las diferentes ciencias no sólo en su especificidad, sino también en las relaciones que entretejen unas con otras. Es por esta razón que el estudio de cada ciencia comienza con el examen de su posición enciclopédica. Así, si en el Cours la sociología es la ciencia final, no sucede lo mismo con el Système, donde la sociología va a verse reemplazada en sus funciones por una séptima ciencia, la moral. Para caracterizar la posición enciclopédica de la sociología, sería necesario considerar las dos ciencias que, respectivamente, la preceden y la siguen en la clasificación. De este modo, nos encontramos con dos parejas familiares: sociología y biología, moral y sociología. Conocemos la importancia fundamental que Durkheim concedía a esta última problemática. Para él, no valdría la pena dedicar ni un minuto de tiempo a la sociología si esta no nos llevase a conclusiones susceptibles de explicar nuestra conducta. Del mismo modo, la constitución de una ciencia de la moral (o ciencia de las costumbres, science des mours o moral sciences) debía ser considerada el resultado del trabajo del sociólogo. Ciertamente, el sentido de este tipo de consideraciones se explica en parte por razones históricas. Así, con la separación de la iglesia y el estado, la Tercera República tenía necesidad de una moral exenta de creencias teleológicas. Pero nos olvidamos una vez más que fue Comte quien indicó el camino. A pesar de que esto se corresponda mal con la idea que hoy tenemos del positivismo, este último había afirmado desde el comienzo y de forma inequívoca su intención de reorganizar la moral. Aunque el análisis de esta cuestión escapa de las posibilidades y de los objetivos de este trabajo, bastará con insistir en que en este caso llegaríamos a la misma conclusión: la sociología durkheimiana se constituyó en parte a través de un diálogo crítico con el pensamiento de Comte. Entre las dos cabe hablar por tanto de continuidad y de ruptura. En esta cuestión, de nuevo, la dependencia con respecto a Comte es evidente, como también es evidente la manera que tiene Durkheim de desarrollar una concepción original. Así, el segundo toma del primero unos conceptos, una temática. Sin embargo, el préstamo no

${ }^{16}$ Sobre esta cuestión, no conviene desatender la parte relativa al vocabulario, y en particular al empleo del término «patología». Hoy en día, el antónimo de «normal» es más bien «anormal». Así, el sentido estadístico se hace más pronunciado y, correlativamente, la connotación moral se hace más débil, sino inexistente. Cuando se habla de una temperatura anormal para una temporada, todo juicio de valor desaparece. Sobre esta cuestión, es interesante ver los remarques de Canguilhem a propósito de la desaparición de lo anormal y sobre los problemas asociados a la pareja anormal-anomalía, (Canguilhem, 1966: 77-85)

EMPIRIA. Revista de Metodología de Ciencias Sociales. N. ${ }^{\circ}$ 16, julio-diciembre, 2008, pp. 43-58. ISSN: $1139-5737$ 
consiste en una simple repetición: se trata más bien de una apropiación libre donde la puesta en marcha de nuevos conceptos es siempre fundamental (cuando se piensa, por ejemplo, en el caso de la moral, en lo que Durkheim tomó prestado de Renouvier y, a través de él, de Kant).

Para quien todavía no conoce la obra de Comte, mi exposición ha sido quizá demasiado fragmentaria. Desde luego, mi objetivo no era presentar directamente su pensamiento sociológico. Al dirigirme a los sociólogos, me parecía necesario en primer lugar establecer aquello que de pleno derecho pertenece a la historia de la disciplina. Para ello, era necesario adoptar un enfoque indirecto y mostrar la influencia profunda que Comte pudo ejercer sobre autores que, como Durkheim, son considerados los padres fundadores de la sociología. Esto es algo que se aprecia ya desde La división du travail social. Extendiendo la división del trabajo más allá de la economía a toda la vida social, Comte creó un concepto clave de la sociología. Sin embargo, el progreso de la división creciente del trabajo, aunque era un factor de progreso representaba para él también una amenaza para la solidaridad. Es precisamente esta conclusión, sobre la que se fundaba la política positiva que Durkheim rechaza, la que la distinción entre solidaridad mecánica y orgánica permite rechazar.

La importancia acordada por el positivismo a la filosofía de la ciencia invita, no obstante, a privilegiar un punto de vista epistemológico. De ahí la elección del capítulo de las Règles de la méthode sociologique, que trata de la distinción entre lo normal y lo patológico. El examen de dichas páginas demuestra que la contribución de Comte a la metodología de la sociología debe ser tenida en cuenta. A menudo, se insiste en su rechazo de las matemáticas sociales ${ }^{17}$. Durkheim había visto perfectamente lo que podía tener de excesivo dicha posición. En este sentido, es la utilización del concepto de media, cuyo origen estadístico es evidente, lo que le permite aportar un criterio objetivo a una distinción que toma prestado de sus antecesores. En lugar de quedarse con este aspecto negativo y desafortunado, hay que quedarse con lo que respecta a las relaciones interdisciplinares de la sociología: Comte nos invita a pensar la ciencia social en sus relaciones no con la psicología o con la economía, sino con la biología, que la precede, y con la moral, que la continúa en la clasificación de las ciencias. Incluso aunque esto haya podido llevar a errores o abusos, la idea es a pesar de todo fecunda.

\section{ANEXOS}

Dado que los textos de Comte son de difícil acceso, me ha parecido útil aportar dos pasajes sobre el principio de Broussais que se encuentran en el Système du Politique Positive y que fueron recogidos por Canguilhem en Le normal et le pathologique.

17 Ver Coumet (2003)

EMPIRIA. Revista de Metodología de Ciencias Sociales. N. ${ }^{\circ}$ 16, julio-diciembre, 2008, pp. 43-58. ISSN: $1139-5737$ 
«La teoría positiva de la modificabilidad de los fenómenos «se condensa enteramente en este principio universal, resultado de la extensión sistemática del gran aforismo de Broussais: toda modificación, artificial o natural, del orden real concierne únicamente a la intensidad de los correspondientes fenómenos..., a pesar de las variaciones de grado, los fenómenos conservan siempre la misma disposición, de modo que todo cambio de naturaleza propiamente dicho, es decir de clase, debe ser considerado contradictorio» ${ }^{18}$.

«La observación juiciosa de las enfermedades instituye, hacia los seres humanos, un experiencia indirecta mucho más adecuada para esclarecer las nociones dinámicas e incluso estáticas que la mayor parte de las experiencias directas. Mi Tratado filosófico ha permitido apreciar la naturaleza y el alcance de dicho procedimiento, del que emanan las principales adquisiciones biológicas. Dicho proceder reposa sobre el gran principio cuyo descubrimiento atribuyo a Broussais puesto que se deriva del conjunto de sus trabajos, pese a que yo me haya limitado a su fórmula general y directa. El estado patológico había sido hasta entonces relacionado con leyes totalmente distintas a las que rigen el estado normal, de tal modo que el análisis de uno no podía aportar nada al conocimiento del otro. Broussais estableció que los fenómenos de la enfermedad coinciden esencialmente con los de la salud, y que sólo se diferencian en su intensidad. Este clarificador principio se ha convertido en la base sistemática de la patología, subordinada de este modo al conjunto de la biología. Aplicado en sentido inverso, el principio sirve para explicar y perfeccionar la conveniencia del análisis patológico en el esclarecimiento de las especulaciones biológicas...Los conocimientos que debemos a dicho principio no puede dar más que una vaga idea de su eficacia posterior. El régimen enciclopédico lo extenderá sobre todo a las funciones intelectuales y morales, a las que el principio de Broussais no ha sido todavía aplicado convenientemente, de tal manera que sus enfermedades nos sorprenden o nos conmueven sin informarnos...además de su eficacia para explicar cuestiones biológicas, este principio constituirá, en el sistema general de la educación positiva, una feliz preparación lógica para procedimientos análogos hacia la ciencia final. Porque el organismo colectivo, en virtud de su complicación superior, comporta problemas todavía más graves, más variados y más frecuentes que los del organismo individual. No tengo ninguna duda de que el principio de Broussais debe ser extendido a dicho dominio, y de hecho yo lo he aplicado a menudo para confirmar o perfeccionar las leyes sociológicas. Pero el análisis de las revoluciones no puede aclarar el estudio positivo de la sociedad sin la iniciación lógica que resulta a este respecto del análisis de los casos más simples que presenta la biología» ${ }^{19}$.

${ }^{18}$ Comte, A. 1851-1854. Système du Politique Positive, T. III, 28, 71. Citado en Canguilhem (1966:19)

19 Comte, A. 1851-1854. Système du Politique Positive, T. I, 28, 651-653. Citado en Canguilhem (1966: 19-20)

EMPIRIA. Revista de Metodología de Ciencias Sociales. N. ํ 16, julio-diciembre, 2008, pp. 43-58. ISSN: $1139-5737$ 


\section{BIBLIOGRAFÍA}

Aron, R. (1998): Les grandes étapes de la pensée sociologique, Paris: Gallimard.

BecQuemont, D. et Muchielli, L. (1998): Le cas Spencer, Paris: P.U.F.

Berthelot, J.M. (2002): «Durkheim lecteur de Comte» en Bourdeau, M. y Chazel, Fr. (éds): Auguste Comte et l'idée de science de l' homme, Paris: L'Harmattan.

Bourdeau, M. (2004): «L'idée de point de vue sociologique», Cahiers internationaux de sociologie, CXVII, pp. 226-238.

Canguilhem, G. (1966): Le normal et le pathologique, Paris: Presses Universitaires de France.

CHAZEL, F. (2004): «Sur quelles bases établir des relations stables entre historiens et sociologues?», Revue européenne des sciences sociales, XLII, n. ${ }^{\circ} 129$, pp. 63-72.

Comte, A (1929) Système du Politique Positive, ou Traité de sociologie instituant la Religion de l'Humanité, 4 vol., 4. . ed, Paris: Société Positiviste (Versión original 18511854).

- (1966): Catéchisme positiviste, Paris: GF.

- (1970): Écrits de Jeunesse, Mouton: Paris.

- (1975): Cours de Philosophie positive, 2 vol., Paris: Hermann.

- (1995): Discours sur l' esprit positif, Paris: Vrin.

- (1998): Discours sur l' ensemble du positivisme, Paris: GF.

COUMET, E. (2003): «El cálculo de probabilidades, aberración radical del espíritu matemático», Empiria, n. ${ }^{\circ}$ 6: Uned, pp. 127-141.

CuIn, C. H. (éd.) (1997): La méthode durkheimienne d'un siècle à l'autre, Paris: PUF.

DAVY, G (1949): «L'explication sociologique et le recours à l'histoire d'après Comte, Mill et Durkheim», Revue de Métaphysique et de Morale, pp. 330-361.

DEWEY, J. (1939): «Unity of science as a social problem», en: O. Neurath (editor): Encyclopedia of Unified Science, Vol. I, n. ${ }^{\circ}$ 1, University of Chicago Press, pp. 29-38.

DURKHEIM, E (1960): Le suicide, Paris: PUF.

- (1973): De la division du travail social: étude sur l'organisation des sociétés supérieures, Paris: PUF.

- (1998): Les règles de la méthode sociologique, Paris: PUF.

GANE, M. (1997): «Durkheim contre Comte», en H. Cuin (éd.) : La méthode durkheimienne d' un siècle à l'autre, PUF.

- (2006): Auguste Comte, Routledge.

GuILlo, D. (2006): «La place de la biologie dans les premiers textes de Durkheim: un paradigme oublié?», Revue Française de Sociologie, n. ${ }^{\circ}$ 3, vol. XLVII.

HeIlbRon, J. (1993): «Ce que Durkheim doit à Comte», en Ph. Besnard, M. Borlandi et P. Vogt (eds) : Division du travail et lien social, PUF, p. 65.

- (2007): «Sociologie et positivisme en France au XIX ${ }^{\mathrm{e}}$ siècle: les vicissitudes de la Société de Sociologie (1872-1874)», Revue Française de Sociologie, (46-2), pp. 307331.

Hubert, R. (1927): Les grands philosophes, Rasmussen: Paris.

LACROIX, J. (1956): La sociologie de Comte, Paris: PUF.

PARSONS, T. (1961): Theories of Society, New York: Glenncoe Press.

VATIN, F. (2005): «A quoi rêvent les polypes?», en: Trois essais sur la genèse de la pensée sociologique, Paris:La découverte.

YAMASHITA, M. (1995): «La sociologie française entre Auguste Comte et Émile Durkheim: Émile Littré et ses collaborateurs», L'année Sociologique, 45. 


\section{RESUMEN}

Este artículo completa un texto que, bajo el título «Ciencia, religión y sociedad en Auguste Comte», fue publicado en el año 2003 en las páginas de esta revista. Después de un breve repaso de los conceptos sociológicos fundamentales del Cours de philosophie positive, se muestra como el pensamiento de Durkheim se constituyó a través de una discusión crítica con el positivismo. Concretamente, se examinan dos momentos fundamentales de dicha confrontación. En primer lugar, se analiza como la distinción que Durkheim establece en La división du travail social (1893) entre solidaridad mecánica y solidaridad orgánica tenía como objetivo fundamental demostrar que Comte se había equivocado al extraer ciertas consecuencias sobre el progreso de la división del trabajo. En segundo lugar, se muestra como en Les Règles de la méthode sociologique (1895), Durkheim atribuye a Comte la distinción entre lo normal y lo patológico y reinterpreta dicha distinción a partir del concepto de «media» de Quételet. En este contexto, concluimos señalando que Durkheim debe a Comte la idea de que la sociología permite construir una ciencia de la moral.

\section{PALABRAS CLAVE}

Patología social, norma y media, división del trabajo, solidaridad, moral y sociología

\section{ABSTRACT}

This article completes a text that, under the title «Ciencia, religión y sociedad en Auguste Comte», was published in this journal in the year 2003. After a brief review of the fundamental sociological concepts of Cours de philosophie positive, it is shown that Durkheim's thinking was constituted through a critical discussion with positivism. Concretely, two fundamental moments of this confrontation are examined. First, we analyse that the distinction Durkheim settles in La división du travail social (1893) between mechanical solidarity and organic solidarity had the fundamental objective to demonstrate that Comte had made a mistake when extracting certain consequences about the work division progress. Second, it is shown that in Les Règles de la méthode sociologique (1895), Durkheim attributes Comte the distinction between normal and pathological, and he reinterprets this distinction starting from Quételet's concept of «average». In this context, we conclude pointing out that Durkheim owes Comte the idea that sociology allows to build a science of morals.

\section{KEY WORDS}

Social pathology, norm and average, work division, solidarity, morals and sociology 